\title{
AN EFFICIENT DATA TRANSMISSION POLICY IN AN INTEGRATED VOICE-DATA DS-CDMA NETWORK
}

\author{
Oriol Sallent, Ramon Agustí \\ Departament de Teoria del Senyal i Comunicacions \\ Universitat Politècnica de Catalunya \\ C/ Jordi Girona, 1 - 08034 Barcelona - Spain \\ E-mail : oriol, ramon a xaloc.upc.es
}

\begin{abstract}
CDMA schemes appear to be very promising access techniques for coping with the requirements of third-generation mobile systems, mainly because of their flexibility. This paper proposes an Adaptive S-ALOHA DS-CDMA access scheme as a method for integrating non-real time (i.e. Internet applications) and real-time (i.e. voice) services, by exploiting the potentials of CDMA under time-varying channel load conditions. The Adaptive component makes data terminals autonomously change their transmission rate according to the total (voice+data) channel occupancy, so that the minimum possible data delay is almost always achieved.
\end{abstract}

\section{I.- INTRODUCTION}

The astonishing growth rates of the Internet and WWW markets in recent years show the enormous potentials of packet-based services. Data traffic figures predicted for third-generation-on mobile communications that might have seemed far too high a few years ago, are now seen as low. However, many of the packet schemes proposed for the third generation are still mainly voice-oriented schemes, especially TDMA-based schemes like PRMA [1] or PRMA ++ [2]. Recently, the proposal in [3] has improved the flexibility of PRMA++, so that it is capable of handling a mix of traffic sources, at the cost of increasing signalling and co-ordination among terminals. Nevertheless, in general, the bursty characteristics of data traffic should yield benefits for access technologies capable of allocating a large amount of simultaneous resources instantly and in a decentralised way, so as to reduce average delay and increase throughput. ALOHA-like techniques, largely used in LAN scenarios, could fulfil these requirements.

Radio resource allocation is also an important issue when designing future cellular systems. However, most of the studies published in this field rely on Fixed Channel Allocation (FCA) and Dynamic Channel Allocation (DCA) of circuit mode-oriented connections [4]. By allowing any cell to allocate any resource, DCA is attractive because it does not need any cellular planning. DCA can use different schemes. One way is to allocate resources exposed to the least interference, as in DECT. Unfortunately, the fast-changing interference environment appearing with bursty packet data prevents the use of least-interference strategies, since interference measurement periods can be similar to, or even higher than, a message transmission. Another way is to choose at random within the free resources. ALOHA-like access techniques could be included in this category and could be retained as appropriate for these scenarios. However, problems such as the high peak values associated with this access when moderate or high bit rates are employed [5], the unfair access results for non-favoured users when capture is introduced and inability to take advantage of full channel capacity when operating below saturation [6], all mean that ALOHA-like techniques are probably not the most suitable.

In this framework CDMA-ALOHA schemes [7, 8] seem very promising ways to deal with the increasing packet-type demand of Internet-like services. CDMA jointly used with ALOHA could retain the advantages of ALOHA without the negative effects of ALOHA mentioned above. In addition, CDMA can be considered a random resource allocation scheme and can also provide real time services, like voice, with the QoS required. Moreover, the resulting ALOHA-CDMA would enable different services to be integrated easily, using natural statistical traffic multiplexing. This property is highly desirable in third-generation systems, since the constant evolution of the applications required generates uncertainty about what these systems will be required for: the integrated scheme will be flexible, whatever the final solution.

Earlier studies of packet CDMA were initially limited to interactive data source models $[8,9]$. On the basis of IS-95, intensive work was done on circuitoriented CDMA for voice applications [10]. The need to integrate both types of traffic generated a lot of interest in this topic. Thus, [11] focused on the effect of FEC and the propagation loss coefficient on network capacity. Approaches considered in [7], [12] and [13] deal with receiver-oriented systems, where subscribers have to share a reduced set of code sequences. However, 
although all of these papers on packet CDMA show the flexibility inherent to CDMA, none of them fully exploits the potentials of CDMA by taking full advantage of time-varying channel load conditions.

This paper will consider an adaptive S-ALOHA CDMA access $[14,15]$ as a strategy for coping with the requirements of both bursty packet and voice transmission. In addition, this technique, which can be decentralised (mobile-controlled), allows us to use the current available capacity of the channel, which varies with the traffic load, so as to decrease the message delay delivery when the traffic load decreases. For this purpose we take advantage of the CDMA's flexibility in bandwidth utilisation, as it allows the change in processing gain. The point is that networks operate below their maximum capacity most of the time, so an improvement in the QoS, in terms of lowering system latency, could be provided for users without the intervention of Control Admission, which is concerned with the maximum number of users admitted [16] or the maximum SIR admitted [17]. In fact, there are scenarios that are not capacity-limited, i.e. those with reduced traffic loads, as in many indoor environments, where SALOHA is optimum because the channel access delay is almost zero and the system is much simpler than other schemes.

The rest of the paper is organised as follows. In section 2 the Adaptive S-ALOHA DS-CDMA scheme is presented in terms of both throughput and delay performance for data only traffic. Section 3 shows that the resulting system is able to integrate voice and data terminals, reducing data transmission delay. Finally, some conclusions close the paper in section 4 .

\section{II.- ADAPTIVE S-ALOHA DS-CDMA}

A BPSK DS-CDMA access system with a processing gain given by $G_{p}$ is discussed. Under a transmitter-oriented approach, all users are assigned their own random PN signature sequence. The network topology consists of a multiple-receiver scheme at the central base station and a number of users located around it, in line with the conventional star architecture that enables up-link and down-link transmission paths to exist on two different frequency bands. Additionally, a perfect power control capable of mitigating fadings of the channel is discussed.

If ideal instantaneous power control is assumed, the channel can be seen as an AWGN if we use the gaussian hypothesis to model the interference caused by other users [18]. Under these conditions, the following expressions hold for the evaluation of the BER :
$P_{b}(n)=Q\left(\sqrt{2 \frac{E_{b}}{N_{o}}}\right) ; \quad \frac{E_{b}}{N_{o}}=\frac{3 G_{p}}{2(m-1)}$

where $n$ is the number of simultaneous users. Then, the probability of correctly detecting a packet containing $L$ bits is :

$$
P_{c}(n)=\left[1-P_{b}(n)\right]^{L}
$$

Keeping the transmission bandwidth constant, and taking as reference a transmission rate of $v$ bits $/ \mathrm{s}$, packet length of $L$ bits and a processing gain $G_{p}$, the transmission rate could be increased by a factor $\alpha(\infty>1)$ at the expense of a reduction in the spread capacity. That is, transmitting at rate $\alpha \nu$ bits/s would allow a processing gain of $G_{l} / \alpha$. However, in exchange for this reduction in processing gain, $\alpha L$ bits per time slot can be allocated, instead of only $L$.

When S-ALOHA DS-CDMA operates below the saturation point, not all the features are used efficiently. In particular, when the channel load is low, a high processing gain is not necessary because there is little interference in the system. One could say that it is the access technique that limits the throughput of the system under low loads instead of what one expects in a CDMA system, which should be interference-limited. Since the need for processing gain (protection against multi-user interference) depends on the channel load, the system performance can be improved by using an adaptive change of the transmission rate algorithm according to the channel load conditions.

Without losing general applicability, we will only consider three different transmission rates in the sequel : $\alpha v$ bits $/ s$ with $\alpha=1,2$ or 4 . Then, this algorithm should be able to maximise the number of correctly received bits for a given packet duration period by selecting the most suitable mobile transmission rate $(4 v$, $2 v, v$ bits $/ s$ ) at any given slot depending on the number of active users (i.e. the interference level).

We now propose a practical solution. The proposed simple algorithm performed by the mobile station (MS) works as follows: each terminal traces its own evolution during the transmission time, i.e. terminals count their successful and erroneous packets. In the absence of errors, the mobile will assume a low traffic load and try to use a higher transmission rate. The throughput should be increased in this way. If errors occur, the mobile decides that the channel is over-loaded and tries a lower transmission rate. In this case, fewer bits per packet are transmitted, but an overall improvement in throughput should follow, because these 
bits can be detected correctly since processing gain increases accordingly $[14,15]$.

For simulations purposes $N=200$ registered users generating messages with arrival rate $\lambda$ (variable that controls the channel load) and average message length $(1 / \mu)=1$ Kbyte have been considered. A packet length of $L=200$ bits and processing gain $G_{p}=127$ have been set for the reference transmission rate $v$ bits $/ s$. Every terminal is assumed to have a FIFO buffer with capacity for storing 20 Kbytes. The system is said to be stable while none of the buffers presents overflow.

Fig. 1 shows the performance attained with the MS algorithm. In spite of the simplicity of the algorithm, the envelope of the three individual graphs $(v, 2 v$ and $4 v$ bits/s) is almost reached. Results have been obtained with max_tr=1 and min_suc=7, which is the best choice.

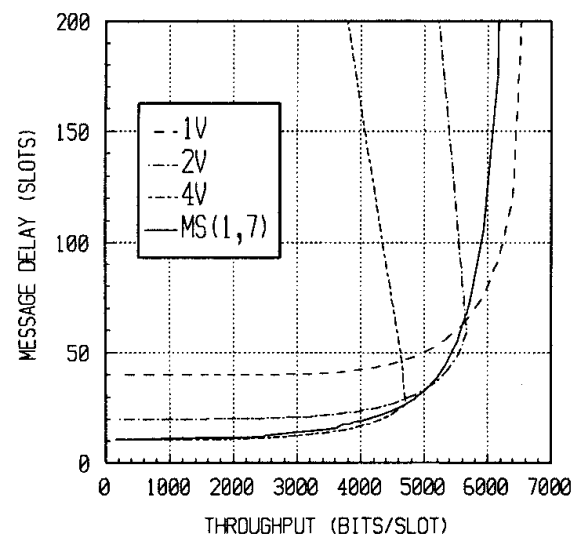

Fig. 1. Performance attained with the MS algorithm.

\section{III.- ADAPTIVE S-ALOHA DS-CDMA FOR VOICE AND DATA MULTIPLEXING}

The random-access operating mode of packet CDMA provides near-perfect statistical multiplexing of any given mix of traffic sources. No complex protocol structures are required in the MAC layer for the integration of circuit-mode and packet-mode traffic. In particular, the proposed adaptive S-ALOHA DS-CDMA scheme is able to handle both real-time and non-real time traffic, e.g. voice and data sources.

A classical speech model is considered, where sources create a pattern of talkspurts and gaps, which are assumed to have statistically independent exponentially distributed duration. The mean duration of the talkspurts and gaps are $1.41 \mathrm{~s}$ and $1.74 \mathrm{~s}$ respectively. This yields a voice activity factor of 0.45 . A $8 \mathrm{Kbps}$ voice encoder is considered. Though S-ALOHA provides a natural protection mechanism, i.e. retransmission, the voice traffic can not take advantage of it due to the high delay constraints, and so lost packets may occur because voice packets are not retransmitted. Experimental measurements for voice quality indicate that a packet loss of $1 \%$ is still acceptable. In order to diminish the number of lost packets due to multi-user interference, voice information is convolutionally encoded with the $\mathrm{R}=1 / 2$ convolutional code with generator polynomial (133 171). The following bound can be established for this code [19] :

$$
P_{e} \leq \frac{1}{2}\left(36 D^{10}+211 D^{12}+1404 D^{14}+11633 D^{16}\right)
$$

$P_{e}$ being the BER after decoding and $D$, for soft decision at the decoder output, is given by :

$$
D=e^{-E_{b} / N_{o}}
$$

Previous results fixed neither the transmission bandwidth nor the time slot duration. At this point, it is necessary to consider some representative values in order to introduce the voice dynamics. Thus, for instance, we could fix a tentative $T_{\text {slot }}=10 \mathrm{~ms}$, which for the reference transmission rate $v$ bits $/ s$ ( $L=200$ bits) with $G_{p}=127$, leads to a bandwidth occupation $B W=2.54 \mathrm{MHz}$. For a voice encoder delivering 160 bits every $20 \mathrm{~ms}$, we can split this block into two 80 -bit packets. These 80 bits are convolutionally encoded, giving 160 bits, and still leaving room for 40 overhead bits (tail bits for Viterbi decoding and some spare bits in case synchronisation is not as ideal as supposed) to complete the 200 bits packet.

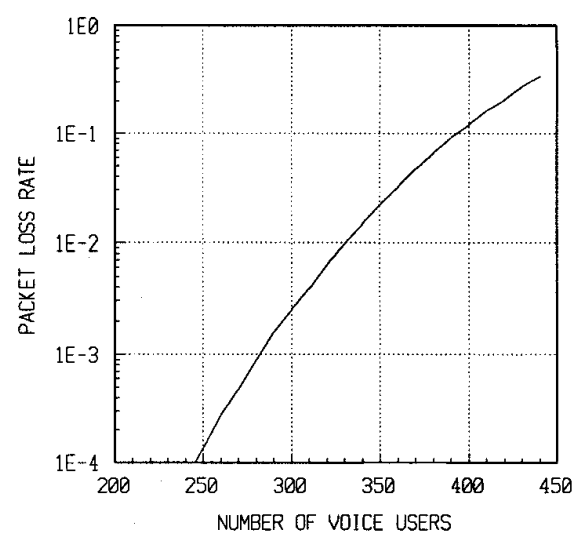

Fig. 2. Packet loss probability for voice-only transmission.

As a first result, Fig. 2 shows the packet loss rate for a voice-only transmission as a function of the 
number of voice users, $N_{v}$. For a $P_{l o s s}=1 \%$ quality threshold we find $N_{v} \approx 330$ users.

Table 1 shows the maximum number of data users that can be supported for different numbers of voice users, $N_{v}$, for $\lambda=0.09$ and $(1 / \mu)=1 \mathrm{Kbyte}$. In this case $(\lambda \mu)=720 \mathrm{bits} / \mathrm{s}$, which corresponds to the $20 \%$ of average load generated by a voice user $(8 \mathrm{Kbits} / \mathrm{s}$ voice encoder with 0.45 voice activity factor). It is worth noting that increasing the transmission rate by a given factor reduces capacity by less than this factor. Additionally, for higher $\lambda$ a lower number of data users can be supported because of the greater activity. In fact, there is an inverse relation between data activity and maximum capacity, as Fig. 3 shows. These results do not include channel coding for data users.

\begin{tabular}{|c|c|c|c|}
\hline $2=0.09 .(1 /)=1 / \mathrm{NO}$ & $N_{\nu}=40$ & $N_{\nu}=60$ & $N_{\nu}=80$ \\
\hline$v$ bits $/ \mathrm{s}$ & 533 & 355 & 182 \\
\hline $2 v$ bits $/ \mathrm{s}$ & 395 & 245 & 140 \\
\hline $4 v$ bits $/ \mathrm{s}$ & 286 & 164 & 74 \\
\hline
\end{tabular}

Table 1. Maximum numbers of data users for different numbers of voice users.

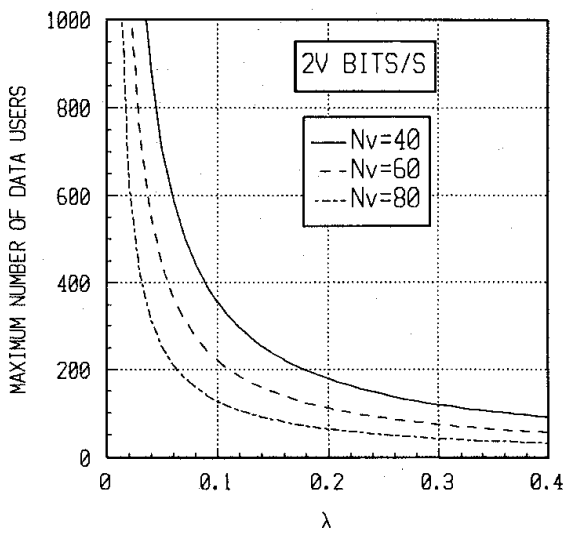

Fig. 3. Impact of data activity on system capacity.

Maximum capacity is not the only pertinent performance measurement, for message delay as a function of the number of registered users also is. Fig. 4 shows the results for data users transmitting at $2 v$ bits $/ \mathrm{s}$ and for the case $\lambda=0.045$ and $(1 / \mu)=1$ Kbyte. Obviously, the lower the number of data or voice users, the lower the minimum message delay will be. As $N_{v}$ increases, the minimum data message delay also increases, since more capacity is devoted to voice transmission.

In the above voice and data integrated framework, the proposed MS algorithm can be applied to data terminals, so the message delivery delay can be reduced when possible (i.e. when there is low load in the system) and consequently system efficiency can be improved. Fig. 5 shows the individual performances for data terminals transmitting at different transmission rates and the performance obtained when the MS algorithm is used. $N_{v}=40$ voice users are served together with the data users. We conclude that the MS is able to operate in a mixed voice-data environment, optimising data transmission.

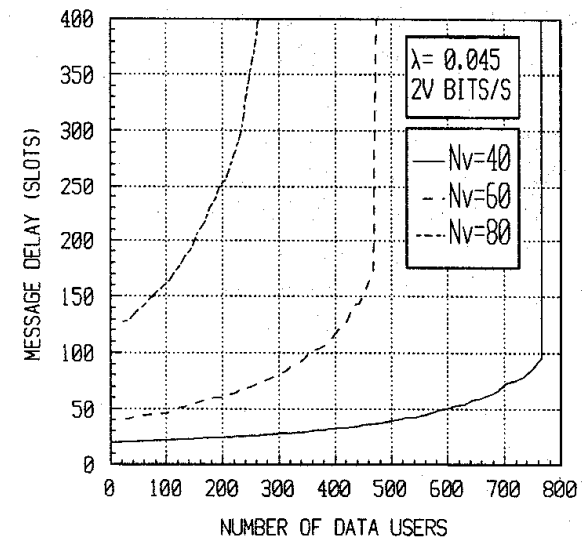

Fig. 4. Data performance for different number of voice users.

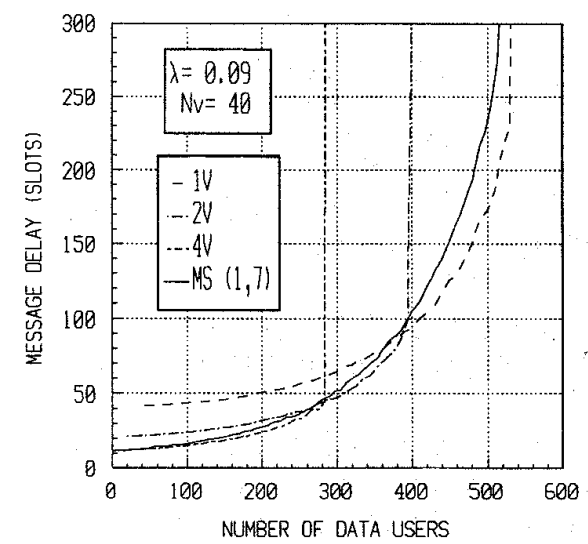

Fig. 5. Data delay behaviour with the MS algorithm.

\section{IV.- CONCLUSIONS}

In this paper we have proposed an Adaptive SALOHA CDMA access technique as a strategy for coping with the requirements of both bursty-packet and voice transmission. The solution is based on CDMA's flexibility in bandwidth utilisation, allowing the change of the transmission rate when for any reason the network is operating below maximum capacity. In fact, in such circumstances, S-ALOHA is the best system for 
handling the reduced traffic load, in the sense that channel access delay is minimum and the system is less complex than other schemes.

\section{ACKNOWLEDGMENTS}

The work described in this paper was carried out within the CICYT TIC94-0870-C02-01 project, in the framework of National Plan of Spain.

\section{VI.- REFERENCES}

[1] S. Nanda, D.J. Goodman, U. Timor, "Performance of PRMA : A Packet Voice Protocol for Cellular Systems", IEEE Transactions on Vehicular Technology, Vol. 40, No. 3, August 1991, pp. 584-598.

[2] J. Devile, J.Dunlop, "Development of the Multiple Access Technique for the ATDMA Air Interface", RACE Mobile Workshop, Metz, 16-18 June 1993, pp. 425-429.

[3] G.Anastasi, D. Grillo, L. Lenzini, "An Access Protocol for Speech/Data/Video Integration in TDMABased Advanced Mobile Systems", IEEE Journal on Selected Areas in Communications, Vol. 15, No. 8, October 1997, pp. 1498-1510.

[4] I. Katzela, M. Naghshineh, "Channel Assignment Schemes for cellular Mobile telecommunication Systems: A Comprehensive Survey", IEEE Personal Communications, Vol. 3, No. 3, June 1996, pp. 10-31.

[5] N. Abramson, "Multiple Access in Wireless Digital Networks", Proceedings of the IEEE, Vol. 82, No. 9, September 1994, pp. 1360-1369.

[6] M. Zorzi, S. Pupolin, "Slotted ALOHA for HighCapacity Voice Cellular Communications", IEEE Transactions on Vehicular Technology, Vol. 43, No. 4, November 1994, pp. 1011-1021.

[7] Z. Liu, M. El Zarki, "Performance Analysis of DSCDMA with Slotted ALOHA Random Access for Packet PCNs “, Wireless Networks 1 (1995).

[8] D. Raychaudhuri, "Performance Analysis of Random Access Packet-Switched Code Division Multiple Access Systems", IEEE Transactions on Communications, Vol. Com-29, No. 6, June 1981, pp. 895-901.

[9] A. Polydoros, J. Silvester, "Slotted Random Access Spread-Spectrum Networks : An Analytical Framework", IEEE Journal on Selected Areas in Communications, Vol. SAC-5, No. 6, July 1987, pp. 989-1002.

[10] A. Salmasi, K. S. Gilhousen, "On the System Design Aspects of CDMA Applied to Digital Cellular and Personal Communications Networks", Proceedings 41st IEEE Vehicular Technology Conference, St. Louis, May 1991.

[11] R. Ganesh et al., "Performance of Cellular Packet CDMA in an Integrated Voice/Data Network",
International Journal of Wireless Information Networks, Vol. 1, No. 3, 1994, pp. 199-222.

[12] N. Guo, S.D. Morgera, P. Mermelstein, "Common Packet Data Channel (CDPC) for Integrated Wireless DS-CDMA Network", IEEE Journal on Selected Areas in Communications, Vol. 14, No. 4, May 1996, pp. 735748.

[13] L. Tan, Q.T. Zhang, "A Reservation RandomAccess Protocol for Voice/Data Integrated SpreadSpectrum Multiple-Access System", IEEE Journal on Selected Areas in Communications, Vol. 14, No. 9, December 1996, pp. 1717-1727.

[14] O.Sallent, R. Agustí, "A Proposal for an Adaptive S-ALOHA Access System for a Mobile CDMA Environment", IEEE Transactions on Vehicular Technology, May 1998.

[15] O. Sallent, R. Agustí, "A Mobile Controlled Algorithm for Improving the Throughput in a SALOHA DS-CDMA System ", Proceedings of the PIMRC'96, October 15-18, 1996, Taipei, Taiwan.

[16] M. Naghshineh, A.S. Acampora, "Design and Control of Micro-cellular Networks with QoS Provisioning for Data Traffic", Wireless Networks 3 (1997), pp. 249-256.

[17] K. Das, S.D. Morgera, "Interference and SIR in Integrated Voice/Data Wireless DS-CDMA Networks A Simulation Study", IEEE Journal on Selected Areas in Communications, Vol. 15, No. 8, October 1997, pp. 1527-1538.

[18] M. B. Pursley, "Performance Evaluation for PhaseCoded Spread-Spectrum Multiple-Access Communication - Part I: System Analysis “, IEEE Transactions on Communications, Vol. Com-25, No. 8, August 1977, pp.795-799.

[19] M.K. Simon, J.K. Omura, R.A. Scholtz, B.K. Levitt, Spread Spectrum Communications, Computer Science Press, EEUU, 1985. 